\title{
Isolated native tricuspid valve endocarditis caused by viridans streptococcus
}

\author{
John Swiston $\mathrm{MD}^{2}$, Stephen D Shafran MD FRCPC ${ }^{1}$, Narmin Kassam MD FRCPC ${ }^{2}$
}

\begin{abstract}
J Swiston, SD Shafran, $\mathrm{N}$ Kassam. Isolated native tricuspid valve endocarditis caused by viridans streptococcus. Can J Infect Dis 2001;12(5):305-307.

The present report describes a case of native tricuspid valve endocarditis caused by viridans group streptococcus in a 43-yearold man who had recently undergone dental extraction. The patient had no history of intravenous drug use, heart disease or right heart catheterization. Although there have been scattered reports of unusual organisms, to the authors' knowledge, this is the first case of viridans group streptococcal endocarditis involving only the tricuspid valve after dental manipulation.
\end{abstract}

Key Words: Tricuspid valve endocarditis; Viridans streptococcus

\section{Endocardite isolée d'une valve tricuspide naturelle, causée par Streptococcus viridans}

RÉSUMÉ : Voici le cas d'un homme de 43 ans, victime d'une endocardite d'une valve tricuspide naturelle, causée par un streptocoque du groupe viridans à la suite d'une extraction dentaire récente. Le patient n'avait pas d'antécédents d'utilisation de drogues injectables, de cardiopathie ou de cathétérisme du cœur droit. Même si la documentation fait état de quelques cas d'infection par des germes inhabituels, il s'agit, d'après les auteurs, du premier cas d'endocardite causée par un streptocoque du groupe viridans, touchant seulement la valve tricuspide à la suite de soins dentaires.

Solated native tricuspid valve endocarditis (TVE) accounts for only $5 \%$ to $10 \%$ of all cases of infective endocarditis $(1,2)$ and is rarely seen in the absence of intravenous drug use (IDU) or intracardiac catheterization or cardiac anomalies $(3,4)$. The present report describes a case of isolated viridans streptococcal TVE after dental manipulation in a nonintravenous drug user without underlying cardiac disease or central venous catheterization. We are not aware of any cases reported in the English literature of isolated native TVE caused by viridans streptococcal species after dental procedures. The expedient identification of patients with TVE in the absence of predisposing factors can be difficult, and thus delay in diagnosis may delay appropriate therapeutic intervention.

\section{CASE PRESENTATION}

A 43-year-old male patient was referred by his family physician with a two-week history of fever, chills, weakness, anorexia and nausea following a dental extraction. Six days before admission, empirical antibiotic therapy with oral metronidazole and penicillin had been initiated for a presumptive oral infection, although the site of extraction was not swollen and only minimally painful. The patient's symptoms improved transiently but recurred two days after antibiotics were initiated. No respiratory symptoms were described. A referral letter from the patient's family physician indicated a normal chest x-ray, normal complete blood count, erythrocyte sedimentation rate of $23 \mathrm{~mm} / \mathrm{h}$ (reference

Divisions of ${ }^{1}$ Infectious Diseases and ${ }^{2}$ General Internal Medicine, Department of Medicine, University of Alberta, Edmonton, Alberta Correspondence: Dr Narmin Kassam, Department of Medicine, University of Alberta, 2E3.23 Walter C Mackenzie Health Sciences Centre, 8440 - 112 Street, Edmonton, Alberta T6G 2R7. Telephone 780-407-7606, fax 780-407-2680, e-mail nkassam@cha.ab.ca Received for publication November 15, 2000. Accepted March 9, 2001 
0 to $15 \mathrm{~mm} / \mathrm{h}$ ), lactate dehydrogenase concentration of $1016 \mathrm{IU} / \mathrm{L}$ (reference 60 to $100 \mathrm{IU} / \mathrm{L}$ ) and alanine aminotransferase concentration of $162 \mathrm{IU} / \mathrm{L}$ (reference 0 to $35 \mathrm{IU} / \mathrm{L}$ ). A heart murmur of grade II/VI at the lower left sternal border was noted. Blood cultures showing Gram-positive cocci were reported from admission. The patient was otherwise healthy, with no previous history of cardiac abnormalities, murmurs or diseases, and no history of IDU.

On examination, the patient was oriented, in no apparent distress and afebrile with normal vital signs. Clinical findings included a normal first and second heart sound with the absence of a third or fourth heart sound, and a grade II/VI midsystolic, high-pitched murmur heard best at the lower left sternal border. The murmur was louder with inspiration. The jugular venous pulsation was not seen. No splinter hemorrhages, Janeway lesions, Osler nodes or conjunctival petechiae were present. Abdominal inspection revealed tenderness to deep palpation in the right lower quadrant with no organomegaly or pulsatile liver. Pulmonary examination was unremarkable. His admission blood work showed a white blood cell count of $8600 / \mathrm{mL}$, hemoglobin level of $15.1 \mathrm{~g} / \mathrm{dL}$, alanine aminotransferase concentration of $169 \mathrm{U} / \mathrm{L}$ and a normal alkaline phosphatase concentration. Electrocardiogram showed no abnormalities, and chest x-ray results were normal. Blood cultures on both admission and day 1 grew viridans streptococci in two of two vials, later confirmed to be Streptococcus mitis. Altogether, three sets of blood cultures taken over one week were confirmed to be positive for viridans streptococci.

On admission, the patient was started on intravenous pencillin 3,000,000 $\mathrm{U}$ every $4 \mathrm{~h}$ and gentamicin $100 \mathrm{mg}$ every $8 \mathrm{~h}$ based on the blood culture results. Because there was only intermediate susceptibility to the penicillin (minimum inhibitory concentration $1.0 \mathrm{mg} / \mathrm{mL}$ ), antibiotic therapy was subsequently modified to intravenous ceftriaxone (minimum inhibitory concentration $0.25 \mathrm{mg} / \mathrm{mL}$ ) $2.0 \mathrm{~g}$ every day and gentamicin $120 \mathrm{mg}$ intravenously every $8 \mathrm{~h}$. Further blood cultures on days 3 and 10 showed no growth. Transesophageal echocardiogram performed on day 4 of admission revealed a sessile 1.1 by $0.8 \mathrm{~cm}$ vegetation attached to the atrial aspect of the anterior tricuspid leaflet, a flail anterior tricuspid leaflet and severe tricuspid regurgitation $(4+)$. The remaining valves were normal. With antibiotic therapy and standard medical care, the patient's symptoms improved while in hospital. The patient's hepatitis C antibody was negative, and his liver enzymes gradually normalized. The patient was transferred to a peripheral hospital where he subsequently received a total of six weeks of ceftriaxone and gentamicin therapy, with repeat blood cultures showing no growth. There was no evidence of gentamicin toxicity at the end of treatment.

After three months of follow-up, the patient remained short of breath on exertion. His repeat transesophageal echocardiogram showed a moderately enlarged right atrium and right ventricle, with $4+$ tricuspid regurgitation. His right and left ventricular wall motion were normal. After consultation with cardiac surgery, the patient underwent tricuspid valve repair due to reduced exercise capacity. After six months of follow-up, the patient was doing well with no surgical complications.

\section{DISCUSSION}

Isolated native TVE is reported to represent only $5 \%$ to $10 \%$ of all cases of infective endocarditis (1). The lower incidence of right-sided endocarditis compared with left-sided endocarditis has been attributed to the lower rate of congenital and rheumatic heart disease affecting the right-sided valves, the lower right heart pressures and the reduced right heart blood oxygen content (1). The most common predisposing factor for right-sided endocarditis is IDU (3). In a recent Canadian review of 135 cases of infective endocarditis, isolated native TVE was found in only $5 \%$ of non-IDU patients compared with $60 \%$ of patients with a history of IDU (2). Other predisposing conditions commonly associated with right-sided endocarditis include congenital heart disease, intracardiac catheterization and, rarely, pacemakers (3-5).

We have described a case of isolated native TVE caused by viridans streptococcus as defined by the Duke criteria (6) in an otherwise healthy patient with no history of IDU and no known underlying cardiac abnormalities. Although the possibility exists that the patient had an undisclosed history of drug use, there was no evidence of IDU on physical examination, and repeated inquiries into past recreational drug exposure were negative. A surrogate marker of IDU, hepatitis $C$ antibody, was negative. It is also possible that the patient had a previously undetected underlying cardiac abnormality. However, the patient's family physician indicated that there were no previous abnormal cardiac findings on physical examination and no history of rheumatic fever or heart disease. Although the prevalence of tricuspid valve abnormalities in the general population is unknown, a retrospective ultrasonographic study of 7000 consecutive cardiac patients by Hubbard et al (7) suggests that abnormalities of the tricuspid valve are rare.

Transient bacteremia, a condition required for the development of bacterial endocarditis, can arise from dental manipulation (3). Sandre and Shafran (2) reviewed 80 episodes of native valve endocarditis and attributed dental infection and/or procedures as the predisposing condition for bacteremia in $16 \%$ of the cases. Their analysis, however, did not differentiate between right- and left-sided cardiac lesions. Nandakumar and Raju (8) recently reviewed 29 cases of isolated TVE in non-IDU patients and described dental abscess as a possible predisposing factor in one case and dental caries in another. Two cases of TVE after dental manipulation in non-IDU patients without underlying cardiac disease have been described in Japan $(9,10)$.

The microbial etiology of endocarditis varies depending on the anatomical location of the lesion and the predisposing factors. Staphylococcus aureus is by far the most common microorganism causing TVE, accounting for 50\% to $80 \%$ of all cases (1). Other pathogens involved in TVE include Streptococcus species, Gram-negative bacilli (espe- 
cially Pseudomonas aeruginosa) and Candida species (2) Although streptococcal species may result in right-sided endocarditis in combination with left-sided endocarditis in patients with a history of IDU or cardiac abnormalities, analysis by Nandakumar and Raju (8) of 29 cases of isolated TVE in non-IDU patients with no cardiac abnormalities found no cases attributable to viridans streptococcus. In two case reports where dental manipulation was described as the predisposing factor for TVE, nonhemolytic streptococcus was implicated as the pathogenic organism in one instance (9) and no organism was identified in the other (10). A 1984 French review of tricuspid endocarditis (11) reported three cases of infection with $S$ mitis in whom the site of entry was not identified. However, the authors did not differentiate whether these cases had isolated TVE or combination of leftand right-sided endocarditis.

Pulmonary rather than cardiac manifestations are usually the predominant clinical features of TVE $(1,3,12)$. Symptoms arising from pneumonia or septic pulmonary emboli from dislodged vegetative material are common findings in TVE (12). Cardiac manifestations are less prominent than in leftsided endocarditis (1). Murmurs of tricuspid regurgitation or a large $\mathrm{V}$ wave in the jugular veins may be present but can often appear only at later stages of the disease (12). Interestingly, the present patient presented with no pleuropulmonary symptoms but did have an audible right-sided systolic murmur.

\section{CONCLUSIONS}

The present case is unique and, therefore, required a high index of clinical suspicion for early diagnosis for a number of reasons. First, isolated native TVE is uncommonly seen in the absence of IDU, intracardiac catheterization or cardiac abnormalities. Second, viridans streptococcus has not previously been reported as the causative agent after dental manipulation in a non-IDU patient with no known cardiac disease. Third, right-sided endocarditis typically presents with pleuropulmonary symptoms. With the absence of the typical features of TVE, this patient's symptoms were initially believed to have been caused by a dental infection, although no signs of oral infection were present. Therefore, definitive treatment for endocarditis was delayed, and the opportunity for a favorable outcome was compromised. Delays in appropriate therapeutic intervention for TVE potentially may have serious sequelae, and therefore, this diagnosis should be considered in all patients with a history of dental manipulation or other sources of bacteremia, even in the absence of IDU or known cardiac abnormalities.

\section{REFERENCES}

1. Chan P, Ogilby J, Segal B. Tricuspid valve endocarditis. Am Heart J 1989;117:1140-6.

2. Sandre RM, Shafran SD. Infective endocarditis: Review of 135 cases over 9 years. Clin Infect Dis 1996;22:276-86.

3. Remetz MS, Quagliarello V. Endovascular infections arising from right-sided heart structures. Cardiol Clin 1992;10:137-49.

4. Rowley KM, Clubb KS, Smith GJ, Cabin HS. Right-sided infective endocarditis as a consequence of flow-directed pulmonary-artery catheterization. A clinicopathological study of 55 autopsied patients. N Engl J Med 1984;311:1152-6.

5. Arber N, Pras E, Copperman Y, et al. Pacemaker endocarditis: Report of 44 cases and review of the literature. Medicine (Baltimore) 1994;73:299-305.

6. Durack DT, Luke AS, Bright DK. New criteria for diagnosis of infective endocarditis: Utilization of specific echocardiographic findings. Duke Endocarditis Service. Am J Med 1994;96:200-9.

7. Hubbard WN, Westgate C, Shapiro LM, Donaldson RM. Acquired abnormalities of the tricuspid valve an ultrasonographic study. Int J Cardiol 1987;14:311-8.

8. Nandakumar R, Raju G. Isolated tricuspid valve endocarditis in non-addicted patients: A diagnostic challenge. Am J Med Sci 1997:314:207-12.

9. Iwama T, Shigematsu S, Kouichi A, et al. Tricuspid valve endocarditis with large vegetations in a non-drug addict without underlying cardiac disease. Intern Med 1996;35:203-6.

10. Fujito T, Inoue T, Akiya $K$, et al. Tricuspid valve infectious endocarditis associated with dental treatment. Acta Cardiol 1997;52:363-9.

11. Rouveix E, Witchitz S, Bouvet E, Friedlander G, Wolff M, Regnier B, Vachon F. Tricuspid infective endocarditis: 56 cases. Eur Heart J 1984;5(Suppl C):111-5.

12. Panidis IP, Kotler MN, Mintz GS, Segal BL, Ross JJ. Right heart endocarditis: Clinical and echocardiographic features. Am Heart J 1984;107:759-64. 


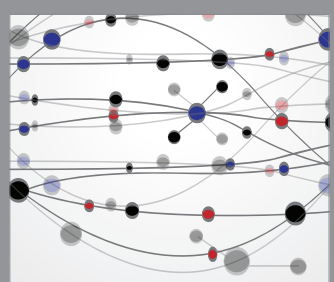

The Scientific World Journal
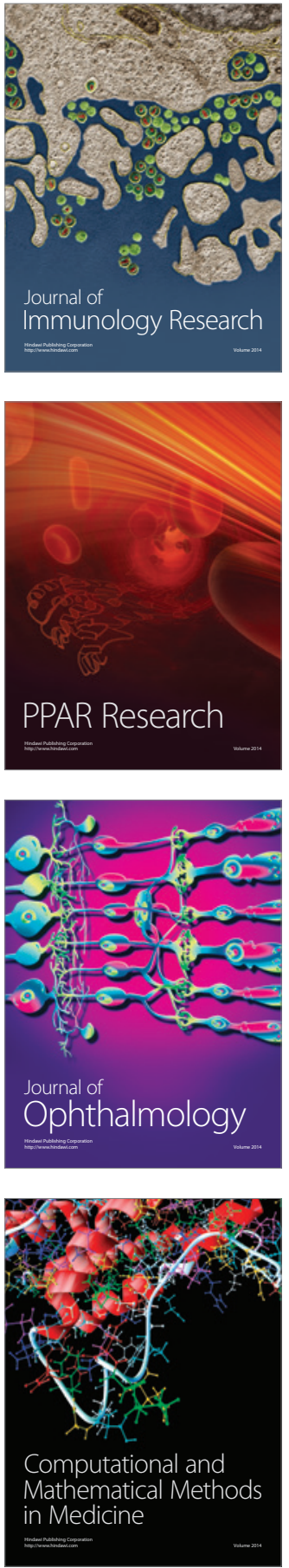

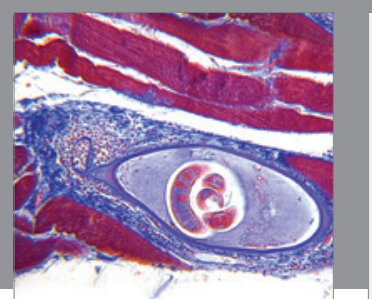

Gastroenterology Research and Practice

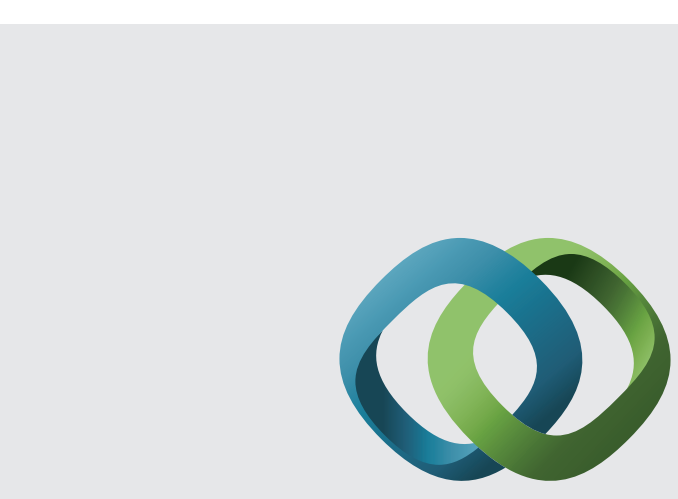

\section{Hindawi}

Submit your manuscripts at

http://www.hindawi.com
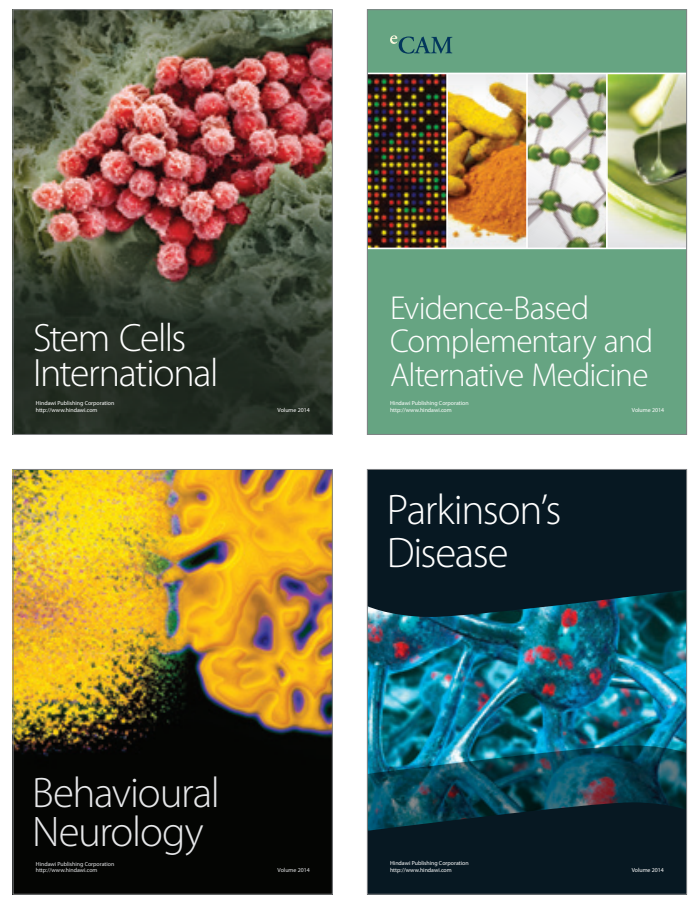
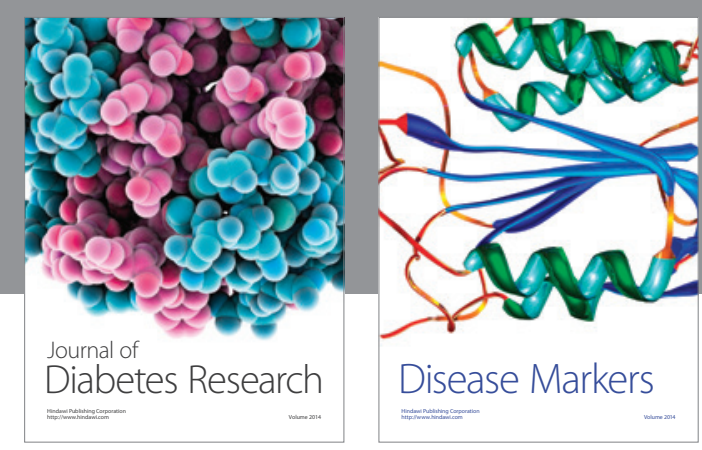

Disease Markers
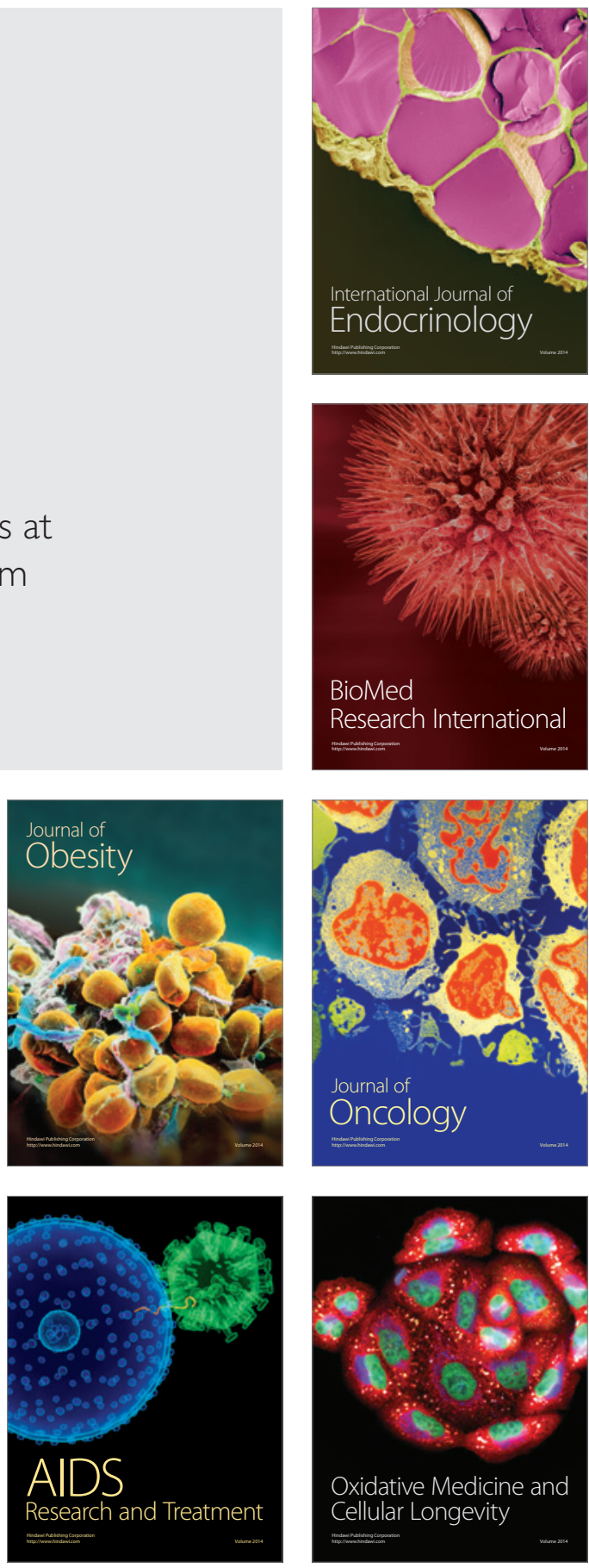